\title{
Entgrenzung der Erwerbsarbeit - Erweiterung der sozialen Sicherheit
}

Die moderne Arbeitswelt ist nicht mehr binär durch Beschäftigung oder Arbeitslosigkeit gekennzeichnet. Viele Menschen nehmen heutzutage im Laufe ihres Erwerbslebens oft mehrere und unterschiedliche Arbeitsverhältnisse wahr. Die Übergänge zwischen diesen sind riskant. Die Arbeitslosenversicherung sichert aber nur das Einkommensrisiko bei Arbeitslosigkeit, und angesichts der Risikovielfalt entspricht die aktive Arbeitsmarktpolitik nicht mehr den aktuellen Anforderungen, etwa dem Risiko einer Erosion der Bildung im Erwerbsverlauf oder dem Risiko unsteter und existenzgefährdender Einkommen durch befristete Beschäftigung und niedrige Löhne. Die Entgrenzung der Erwerbsarbeit bedarf daher neuer sozialer Sicherheiten, um die Exklusionsgefahren am Arbeitsmarkt einzudämmen.

\section{Entgrenzung der Erwerbsarbeit}

Wer noch vor zwei Jahren das Klagelied der zunehmenden Entgrenzung der Arbeit angestimmt hätte, wäre in Deutschland kaum auf Widerspruch gestoßen. Jetzt, nach einem Jahr, in dem nahezu eine halbe Million regulärer Arbeitsplätze geschaffen wurde, stößt eine solche Melodie auf weniger Resonanz. Aber umgekehrt wird kein Schlager daraus, wie gleich zu demonstrieren sein wird. Wenn Wissenschaft überhaupt einen Beitrag zur Generalisierbarkeit von Ent- wicklungen leisten kann, dann ist es ihre Fähigkeit, einen analytischen Blick zurück und über den nationalen Tellerrand hinaus zu werfen. Deshalb soll diesem Essay ein kleiner vergleichender Exkurs zum Wandel der Erwerbsbeteiligung in Deutschland vorangestellt werden.

Der Verlauf der breiten Erwerbsquote ${ }^{1}$ in Deutschland seit 1985 (Tabelle 1) zeigt, dass ein Trend zur Entgrenzung der Erwerbsarbeit tatsächlich vorhanden ist. Er ist jedoch bei Weitem nicht so dramatisch, wie er an die Wand gemalt wird. In den letzten 20 Jahren hat das Niveau der Beschäftigung im sogenannten Normalarbeitsverhältnis - gemessen an der gesamten erwerbsfähigen Bevölkerung - leicht abge-

\begin{tabular}{|c|c|c|c|c|}
\hline Frauen und Männer & 1985 & 1997 & 2005 & Trend \\
\hline Breite Arbeitslosenquote & 5,7 & 9,3 & 9,8 & + \\
\hline 1. registrierte Arbeitslose & 5,4 & 7,8 & 8,8 & + \\
\hline 2. Teilnehmer in Maßnahmen² & 0,3 & 1,5 & 1,0 & $+/-$ \\
\hline Teilzeitbeschäftigungsquote & 7,1 & 11,3 & 15,0 & ++ \\
\hline 3. Teilzeit $<15 \mathrm{~h} /$ Woche & 1,5 & 3,1 & 5,5 & ++ \\
\hline 4. Teilzeit $>15 \mathrm{~h} /$ Woche & 5,6 & 8,2 & 9,5 & + \\
\hline Selbstständigenquote 3 & 7,3 & 6,2 & 7,6 & $-1+$ \\
\hline 5. Selbstständige in Teilzeit & 2,3 & 0,7 & 1,2 & $-/+$ \\
\hline 6. Selbstständige in Vollzeit & 5,0 & 5,5 & 6,4 & + \\
\hline Breite Vollzeiterwerbsquote & 47,9 & 46,3 & 43,8 & - \\
\hline 7. Zeitarbeit & 0,1 & 0,4 & 0,8 & ++ \\
\hline 8. befristete Beschäftigung & 2,0 & 2,5 & 3,1 & ++ \\
\hline 9. Auszubildende & 3,6 & 2,7 & 2,9 & $-/(+)$ \\
\hline 10. Beamte und Soldaten & 5,2 & 3,3 & 3,1 & - \\
\hline 11. Im "Normalarbeitsverhältnis" & 37,0 & 37,4 & 33,9 & - \\
\hline Breite Erwerbsquote 4 & 68,0 & 73,0 & 76,2 & + \\
\hline \multicolumn{5}{|c|}{$\begin{array}{l}\text { 1) Gemessen in \% der erwerbsfähigen Bevölkerung im Alter von } 15 \text { bis } 64 \text { Jahre. } \\
\text { 2) Personen, die bei der Arbeitsagentur gemeldet sind, aber nicht den Status als Arbeitslose haben; die meisten dieser gemeldeter } \\
\text { Personen nehmen vermutlich an arbeitsmarktpolitischen Maßnahmen teil oder warten darauf, teilzunehmen. Eingeschlossen sind } \\
\text { aber nicht Personen in anderen Kategorien des Arbeitsverhältnisses, zum Beispiel subventionierte Beschäftigte in privaten Unter- } \\
\text { nehmen oder Teilnehmer in befristeten Arbeitsbeschaffungsmaßnahmen; diese gelten statistisch als beschäftigt. } \\
\text { 3) Ein steigender Anteil der Teilzeitbeschäftigten arbeitet auch in befristeten Arbeitsverhältnissen: } 1991 \text { war die Quote der befris- } \\
\text { teten Teilzeitbeschäftigten } 0,8 \%, 2005 \text { betrug sie schon } 1,8 \% \text {. } \\
\text { 4) Die Summe der Kategorien } 1 \text { bis } 11 \text {. }\end{array}$} \\
\hline \multicolumn{5}{|c|}{ Quelle: Mikrozensus und eigene Berechnungen; Oschmiansky (2007). } \\
\hline
\end{tabular}

nommen. Seit 1985 ist die Beschäftigungsquote im Normalarbeitsverhältnis von $37 \%$ auf 33,9 \% gesunken. Mit anderen Worten: Nur noch ein Drittel der erwerbsfähigen Bevölkerung befindet sich in einem unbefristeten und abhängigen Vollzeitbeschäftigungsverhältnis. Davon abweichende Arbeitsverhältnisse - befristete Beschäftigung, Leih- oder Zeitarbeit, Solo-Selbstständige und vor allem Teilzeitarbeit haben zugenommen. Rechnet man als Erwerbsverhältnis noch die gestiegene Arbeitslosigkeit und die Menschen in arbeitsmarktpolitischen Maßnahmen hinzu, dann ist die breite Erwerbsquote von $68 \%$ auf $76,2 \%$ angestiegen.

Stünden die neuesten Zahlen für das Jahr 2007 in dieser Differenzierung schon zur Verfügung, könnten wir sicher sein, dass die breite Erwerbsquote noch weiter gestiegen ist. Aufgrund des Konjunkturaufschwungs könnten wir auch feststellen, dass die sogenannten Normalarbeitsverhältnisse wieder an Gewicht zugenommen haben. Das Fazit dieses Rückblicks ist also weniger, dass die Erosion des Normalarbeitsverhältnisses fortschreitet. Die zentra-

\footnotetext{
1 Die breite Erwerbsquote misst den Anteil aller Erwerbspersonen (einschließlich Arbeitsloser, Teilnehmer in arbeitsmarktpolitischen Maßnahmen, Auszubildender) an der Bevölkerung im erwerbsfähigen Alter (15-64 Jahre).
}

Günther Schmid, Prof. Dr., Direktor der Abteilung Arbeitsmarktpolitik und Beschäftigung am Wissenschaftszentrum Berlin von 1989 bis März 2008 und Professor Emeritus für Ökonomische Theorie der Politik an der Freien Universität Berlin e-mail: gues@wzb.eu 
Zunahme atypischer Arbeitsverhältnisse einhergeht.

Zweitens können, wie das Zusammenclustern sozialdemokratischer und liberaler Beschäftigungssysteme zeigt, Arbeitsund Sozialschutz flexibler Beschäftigungsverhältnisse in Europa offensichtlich ganz unterschiedlich ausgeprägt sein. Zwar sind die Risiken in solchen Beschäftigungsverhältnissen höher als im sogenannten Normalarbeitsverhältnis; in einem flexiblen Beschäftigungsverhältnis zu sein, bedeutet aber nicht automatisch Rechtlosigkeit im Arbeits- und Sozialschutz. Damit stellt sich die Frage, wie parallel zur Entgrenzung der Erwerbsarbeit die soziale Sicherheit erweitert werden könnte.

\section{Möglichkeiten der Erweiterung sozialer Sicherheit}

\subsection{PFLICHTVERSICHERUNG, GRUNDSICHERUNG UND GEWINNBETEILIGUNG}

Im Rahmen dieses Beitrages müssen ausgewählte Beispiele genügen. Beginnen wir mit der neuen Selbstständigkeit, also dem europaweiten Trend zur Solo-Selbstständigkeit. Diese ist mit spezifischen Risiken verbunden: mit dem Risiko unregelmäßiger und häufig niedriger Einkommen, dem Risiko der Auftragslosigkeit in Analogie zur Arbeitslosigkeit und dem entsprechenden Risiko mangelnder sozialer Absicherung vor allem im Alter. Mit der Begrenzung der Pflichtversicherung auf wenige Sondergruppen unter den Selbstständigen stellt Deutschland im europäischen Vergleich eine Besonderheit dar. Denn in der Mehrzahl der europäischen Länder werden alle Selbstständigen durch die staatlichen Pflichtversicherungssysteme erfasst, und in den Niederlanden widmet sich sogar eine Gewerkschaft speziell der Interessenvertretung von Selbstständigen (Schulze Buschoff 2007).

Selbst ein Blick nach Amerika, wo vor einiger Zeit Schauspieler und Drehbuchautoren streikten, ist hier lehrreich. Abgesehen davon, dass die amerikanischen Gewerkschaften ausgerechnet in diesem Bereich stark organisiert sind, würde es kein amerikanischer Fernsehsender wagen, seinen Regisseuren, Schauspielern und Auto- ren Verträge vorzulegen, wie sie in Deutschland üblich sind. Vor allem die Praxis des „Buyout“, d.h. des Ausverkaufs aller Rechte, macht die kreative Fernseharbeit in Deutschland $\mathrm{zu}$ einer unterbezahlten Dienstleistung. In den USA bekommen alle Beteiligten Anteile am Weiterverkauf ihrer Produkte. Das motiviert Talente und schafft in diesem Fall nicht nur mehr, sondern auch bessere Arbeitsplätze.

Das Beispiel des Künstler- und Medienarbeitsmarkts lehrt noch mehr (Schmid 2002, S. 200-216). Es zeigt, dass moderne Instrumente der Arbeitsmarktpolitik nicht unbedingt auf den Arbeitsmarkt im engeren Sinne begrenzt sein müssen. Sie berühren auch den Güter- und Dienstleistungsmarkt. Wo Arbeitsleistungen nicht unmittelbar bewertet werden können, weil der Wert der Leistung ungewiss ist und möglicherweise erst nach zehn Jahren entdeckt wird, muss der Lohn der Arbeit eben auch diese Risiken abdecken - sowohl im negativen als auch im positiven Bedeutungssinn von Risiko. Im negativen Sinne ist Risiko Verlustgefahr, im positiven Sinne Gewinnchance.

Steigt also die Gefahr unregelmäßiger Einkommen, muss die Grundsicherung im Alter teilweise von der Erwerbsbiografie abgekoppelt werden. Daraus folgt, die Vorsorge für das Alter staatlich zu unterstützen, wenn das laufende Erwerbseinkommen dafür nicht ausreicht $-z$. B. durch die Riesterrente für alle, also auch für neue Selbstständige, und durch Beitragskredite für Geringverdiener und Arbeitslose. Steigt die Chance zur zukünftigen Verwertbarkeit der Arbeitsleistungen, müssen z. B. die Autorenrechte gestärkt und Arbeitnehmer an Kapitalerträgen beteiligt werden.

Kommen wir zu den befristeten Arbeitsverhältnissen. Hier soll nur das heiß diskutierte Thema des Arbeitnehmerverleihs oder der Zeitarbeit aufgegriffen werden. Deutschland hinkt in der Entwicklung dieser Beschäftigungsverhältnisse gegenüber vielen Nachbarländern hinterher (Storrie 2002). In der deutschen Diskussion werden auch die Risiken dieser Form von Arbeit stärker als die Chancen hervorgehoben. Je nachdem, von welcher Seite man diese hybriden Arbeitsverhältnisse betrachtet, zeigen sie aber sowohl Licht- wie auch Schattenseiten.

Durch die positive Brille gesehen können Zeitarbeitsfirmen die Auftragsrisiken von Firmen poolen und so prinzipiell zwar keine Arbeitsplatzsicherheit, aber Beschäf- tigungssicherheit bieten. Darüber hinaus können Zeitarbeitsfirmen auch Einstellungsrisiken der Firmen übernehmen und noch unerfahrenen jugendlichen Erwachsenen vielfältige Berufserfahrungen vermitteln und so den Übergang in ein festes Beschäftigungsverhältnis unterstützen. Der niederländische Sozialwissenschaftler Jelle Visser hat in diesem Zusammenhang einmal mit der Feststellung provoziert: „Zeitarbeitsunternehmen sind die Gewerkschaften von morgen." 2

Auf der anderen Seite nutzen Erwerbspersonen, insbesondere jüngere qualifizierte Berufsanfänger, Zeitarbeit als strategisches Mittel zur Berufsplanung. Das gilt etwa für Ingenieure, die von Projekt zu Projekt ziehen, so unterschiedliche Erfahrungen sammeln und damit ihre Beschäftigungs- und Wettbewerbsfähigkeit auf dem Arbeitsmarkt stärken. Viele Zeitarbeitsfirmen haben auf den Fachkräftemangel reagiert und lassen geeignete Kandidaten schulen, damit sie genau auf die angeforderten Profile passen. So lernen etwa Ingenieure spezielle Computerprogramme, die sie für ein bestimmtes Projekt brauchen.

In Berlin gibt es beispielsweise rund 1.000 Zeitarbeits- und Personalvermittlungsfirmen, und die Zahl der Zeitarbeitskräfte hat sich in nur vier Jahren von 10.865 (2003) auf 23.323 (2007) mehr als verdoppelt. Neben Ingenieuren sind vor allem Buchhalter mit Englischkenntnissen, examiniertes Pflegepersonal und Fachkräfte für Callcenter gefragt. Zeitarbeitsfirmen berichten, im Durchschnitt bleibe ein Drittel beim entleihenden Betrieb „kleben“. Bei qualifizierten Kräften sei dieser Effekt eindeutig höher mit steigendem Trend. Zeitarbeit ist allerdings stark konjunktursensibel: 2006 wurden $75 \%$ der Belegschaftserweiterungen über Zeitarbeit abgewickelt, 2007 waren es nur noch $25 \%$ (Martens 2008).

\subsection{MINDESTSTANDARDS UND MINDESTLÖHNE}

Durch die negative Brille gesehen sind aber auch viele schwarze Schafe unter den Unternehmen nicht zu übersehen, die Zeitarbeit zum Lohndumping und zur Ausbeutung durch schlechte Arbeitsbedingun-

\footnotetext{
2 Persönliche Erfahrung auf einer Podiumsdiskussion anlässlich einer Tagung der "Society for the Advancement of Socio-Economics (SASE) " in Amsterdam, Juli 2004
} 
gen ausnutzen (Promberger et al. 2006a, 2006b). Da Zeitarbeitsfirmen mittlerweile weltweit agieren, besteht daher dringender Bedarf an einer wenigstens europaweiten Regulierung von Mindeststandards. Um die Vorteile dieser neuen Flexibilität mit den notwendigen neuen Sicherheiten zu verbinden, müssen vor allem das Gleichbehandlungsprinzip und das Recht auf Festanstellung nach mehreren Arbeitseinsätzen gesetzlich verankert werden. Vorbildhaft sind auch höhere Sozialbeiträge für Zeitarbeit in Frankreich und die Pflicht in den Niederlanden, Lohnbestandteile für Zeitarbeitnehmer in einem Weiterbildungsfond anzulegen.

Zur arbeitsmarktpolitischen Begrenzung der Zeitarbeit gehört schließlich auch die Verankerung eines gesetzlichen Mindestlohns. Darüber hinaus sind andere und übergeordnete Gesichtspunkte für eine solche Begrenzung der Erwerbsarbeit zu nennen. ${ }^{3}$ Dafür spricht an erster Stelle die normativ-ethische Betrachtung: Lohneinkommen für Vollzeitbeschäftigte ohne eingeschränkte Erwerbskapazität sollten nicht unter das verfassungsrechtlich garantierte Existenzminimum fallen. Die Garantie eines Mindesteinkommens - etwa durch Kombilöhne - reicht nicht aus, wenn man das Grundgesetz so interpretiert, dass es auch das Recht zu einer autonomen Lebensführung unabhängig von politischer Willkür garantiert.

Aus ökonomischer Sicht sprechen drei Gründe für einen Mindestlohn. Erstens schützen Mindestlöhne Betriebe vor Schmutzkonkurrenz. Angesichts der räumlichen Entgrenzung von Erwerbsarbeit (Internationalisierung von Arbeitsmärkten) ist dies auch für Unternehmen ein anerkannter und zunehmend bedeutsamer Aspekt. Zweitens wirken Mindestlöhne der Segmentierung von Arbeitsmärkten entgegen, ein Punkt, der weiterer Erläuterung bedarf. Haben Unternehmen Marktmacht (die Theorie spricht dann von einem Monopson), dann können sie den Preis der Arbeit unter das Marktgleichgewicht drücken. Ein Mindestlohn muss deshalb nicht weniger Beschäftigung bedeuten, wenn er lediglich die überdimensionierte Gewinnspanne der Monopsonisten abschöpft. Je segmentierter der Arbeitsmarkt ist, desto dürftiger wird das für einen einzelnen Arbeitnehmer relevante Angebot an Jobs. In solchen „ausgedünnten“ Segmenten des Arbeitsmarkts können auch kleinere Unternehmen an Marktmacht gewinnen.
Wenn sich neben der Abschöpfung von Gewinnspannen noch das Arbeitsangebot aufgrund steigender Löhne erhöht, dann kann ein Mindestlohn sogar beschäftigungssteigernde Wirkung haben, wie einige Untersuchungen etwa in den USA zeigen. Wenn ein Mindestlohn jedoch über dem Gleichgewichtslohn liegt, dann hat er in der Tat beschäftigungsschädliche Wirkungen. Diese differenzierte Sichtweise erklärt, warum empirische Untersuchungen immer wieder $\mathrm{zu}$ anscheinend widersprüchlichen Ergebnissen zur Wirkung von Mindestlöhnen gelangen. Drittens üben wohl dosierte Mindestlöhne auf Betriebe einen Anreiz aus, Produktivität und Qualität von Serviceleistungen zu erhöhen. Davon profitieren wiederum die Konsumenten und die gesamte Gemeinschaft.

Schließlich kommen noch zwei politisch-institutionelle Argumente hinzu: Die Sozialpartner sind, aus welchen Gründen auch immer, nicht mehr in der Lage, aus eigener Kraft einen effektiven nationalen Mindestlohn zu garantieren. Umgekehrt wird jedoch ein Schuh daraus: Wird der Mindestlohn faktisch von den stärksten Gewerkschaften ausgehandelt und staatlich allgemein verbindlich erklärt, dann geht das auf Kosten von Arbeitsplätzen für Arbeitslose und Geringqualifizierte. Vieles spricht daher für einen gesetzlichen SockelMindestlohn, dessen Höhe - wie in Großbritannien - durch eine politisch unabhängige, paritätisch besetzte Kommission vorsichtig austariert werden könnte.

\subsection{STABILISIERUNG VON ERSATZ- UND RENTENEINKOMMEN}

Noch ein Wort zur Entgrenzung der Erwerbsarbeit in Form von Teilzeit. ${ }^{4}$ Die Beschäftigung in Teilzeit entspricht ja keinesfalls mehr dem gewohnten Umfang von 20 Stunden. Sie kann von null Stunden denkt man an das beliebte Blockmodell der Altersteilzeit - bis zu 34 Stunden variieren. Ein Zurück zur 40-Stundenwoche als Regel ist weder realistisch noch wünschenswert. Aber auf der Seite der sozialen Sicherheit ist diese flexible Beschäftigungsform in Europa und insbesondere auch in Deutschland noch nicht ausbalanciert. Immerhin, und da ist Deutschland mit den Niederlanden an vorderster Front, bestehen mit dem Teilzeit- und Befristungsgesetz von 2001 ein Recht auf Arbeitszeitverkürzung und ein Rückkehrrecht auf Vollzeit. Das war heiß umstritten, hat sich in der Zwischenzeit je- doch bewährt. Auch die Bestandsschutzsicherung bei Arbeitslosigkeit ist positiv zu erwähnen.

In der Sicherung der Risiken gibt es jedoch noch erhebliche Defizite. Das betrifft vor allem die unsteten Einkommensströme im Erwerbsverlauf und mangelnde Einkommen im Alter, die mit Übergängen von Vollzeit zu Teilzeit und umgekehrt verbunden sind. Zunächst ist auf die mangelnde Koordination derzeitiger Altersteilzeit mit dem Steuer- und Sozialversicherungsrecht hinzuweisen. ${ }^{5}$ Auch die Anreize für geringfügige Beschäftigung (Mini- und Midijobs) sind teilweise falsch gesetzt; sie werden vor allem von Frauen wahrgenommen, deren Defizite in der Altersvorsorge damit vorprogrammiert sind. ${ }^{6}$

Darüber hinaus sollen hier noch zwei weitergehende Überlegungen zur Diskussion gestellt werden: Erstens die Idee flexibler Rentenanwartschaften, zweitens die Einbeziehung unfreiwilliger Teilzeit in die Arbeitslosenversicherung. Mit flexiblen Rentenanwartschaften würde man während der Vollzeitbeschäftigung Anwartschaften (also Eckpunkte) erwerben, welche die verminderten Anwartschaften bei

3 Vgl. hierzu u.a. Bosch/Weinkopf (2006); Manning (2003); Metcalf (2007) und die jüngsten Diskussionsbeiträge aus verschiedener wie kontroverser Sicht im ifo Schnelldienst (2008).

4 Ausführlicher hierzu Schmid (2008a), S. 103-109 und S. 196-202 mit weiteren Literaturverweisen.

5 Die Einkommen während der zumeist sechsjährigen Altersteilzeit werden zwar in der Regel auf über $80 \%$ des vorhergehenden Lohns aufgestockt, aber nur auf $50 \%$ des ursprünglichen Gehalts sind Steuern und Sozialversicherungsbeiträge zu entrichten, mit Ausnahme der Rentenversicherung. Die notorisch unterfinanzierte gesetzliche Kranken- und Pflegeversicherung erhält somit den halben ursprünglichen Beitrag, die Leistung bleibt aber bei $100 \%$; die Gesamtheit der Versicherten wie Steuerzahler subventioniert hiermit die Altersteilzeitbeschäftigten jährlich mit etwa $900 €$.

6 Bisher nutzt noch nicht einmal ein Drittel der Berechtigten die Riester-Förderung, obwohl Durchschnittsverdiener mit dieser Förderung in $30 \mathrm{Jah}$ ren einen privaten Rentenstock von $120.000 €$ bis $160.000 €$ aufbauen können. Für Geringverdiener ist es nahezu illusorisch, die Rentenkürzungen mit Wirkung von 2001 durch private Vorsorge auszugleichen. Eine Einzelhandelskauffrau müsste z. B. bis zu einem Drittel ihres Einkommens in Vorsorge investieren, um diese Kürzungen auszugleichen. Benachteiligend für Frauen kommt hinzu, dass die Anwartschaft auf betriebliche Altersvorsorge immer noch fünf Jahre beträgt, und erworbene Ansprüche können bei Betriebswechsel immer noch nicht mitgenommen werden, obwohl dies die EU-Kommission schon seit Längerem moniert (Kull/Riedmüller 2007). 
Teilzeit oder gar bei einem Sabbatical ausgleichen würden. Die Bundesanstalt für Angestelltenversicherung hat dazu vor ein paar Jahren realistische Modellrechnungen vorgelegt (Thiede 2000).

Im Zusammenhang mit der Absicherung unsteter Einkommensströme ist auf die bisher wenig beachtete Funktionsweise des Arbeitslosenversicherungssystems in Dänemark hinzuweisen. In diesem immer wieder als Vorbild zitierten Land sind Vollzeitbeschäftigte nicht nur gegen Arbeitslosigkeit abgesichert, sondern auch gegen Teilzeitarbeitslosigkeit im Falle unfreiwilliger Teilzeitbeschäftigung oder im Falle der Arbeitszeitverkürzung wegen Kindererziehung oder Weiterbildung.

Die Generalisierung dieser Beispiele weist auf eine weitergehende Möglichkeit hin, Flexibilität und Sicherheit nicht nur rhetorisch im - mittlerweile weitgehend entleerten und beliebig verwendeten Kunstwort „Flexicurity“ zu verbinden, sondern der Verknüpfung auch inhaltlich einen soliden institutionellen Boden zu geben. Wie also könnte ein positives Verhältnis, sozusagen eine „glückliche Heirat", zwischen diesen beiden unterschiedlichen Seiten des Arbeitsmarkts zustande kommen?

\section{Von der Arbeitslosen- zur Beschäftigungsversicherung}

Die Beantwortung der vorgenannten Frage soll am Beispiel der Arbeitslosenversicherung erläutert werden. ${ }^{7}$ Es besteht kein Zweifel, dass die Neuorganisation der Grundsicherung („Hartz-IV“) ein Gerechtigkeitsdefizit hinterlassen hat. Es ist jedoch $\mathrm{zu}$ bezweifeln, dass die jetzt beschlossene Verlängerung des Arbeitslosengeldbezugs an der richtigen Stelle ansetzt und ausreicht, auf dem Arbeitsmarkt der Zukunft sowohl Gerechtigkeit als auch Effizienz nachhaltig zu gewährleisten. Mit Blick auf die Prinzipien des modernen Sozialstaates kann sogar eher von einem Rückschritt gesprochen werden. Warum?

Es hätte eine Alternative gegeben. Das wäre allerdings nicht der aus der ökonomischen Debatte kommende Vorschlag gewesen, die Arbeitslosenversicherung zu privatisieren und in ein individuelles Beschäftigungskonto zu überführen (Boss et al. 2007). Dieser Idee liegt eine Überbetonung des sogenannten moralischen Verhaltens- risikos zugrunde, d.h. der Gefahr, dass Versicherte das System ausnutzen. ${ }^{8}$ Weiterhin lassen diese Überlegungen unberücksichtigt, dass Arbeitslosigkeit überwiegend unfreiwillig ist, und dass die Risiken häufig stark korrelieren. Eine solidarische Arbeitslosenversicherung ist daher auch im modernen Sozialstaat unverzichtbar. Aber dieses Solidarsystem könnte in der Tat durch eine individuelle Komponente ergänzt werden. Wie könnte diese aussehen? Und wie könnte das Effizienz- und Gerechtigkeitsproblem besser gelöst werden als durch eine bloße Verlängerung des Arbeitslosengeldes für eine spezifische Gruppe?

\subsection{INDIVIDUELLE VERHALTENS- RISIKEN (AN)ERKENNEN}

Die Antwort auf diese Frage erschließt sich mithilfe einiger theoretischer Ansätze, die hier nur knapp skizziert werden können. ${ }^{9}$ Nach der Theorie des sozialen Risikomanagements gilt es, eine Balance zwischen dem moralischen und dem innovativen Verhaltensrisiko herzustellen. Versicherungen haben nicht nur negative, sondern immer auch positive Anreizfunktionen. Sie fördern neben der moralischen Versuchung auch die Bereitschaft, riskante Entscheidungen zu treffen. Eine solche Förderung ist sinnvoll, weil die meisten Menschen - aus welchen Gründen auch immer - eher risikoscheu als risikofreudig sind. Die soziologische Risikotheorie, etwa von Ulrich Beck (1986), betont zu Unrecht immer nur den Gefahrenaspekt von Risiko. Vielleicht passt das zu uns Deutschen, macht das Argument aber keineswegs stärker. Die Kehrseite des Risikos ist die Chance: „Wer wagt, gewinnt.“

Das belegen Theorien aus verschiedenen Disziplinen. Die historische Forschung (Bernstein 1996) verweist auf die zentrale Rolle der Versicherungen bei der Entstehung des Kapitalismus. Ende des 17. Jahrhunderts emanzipierten sich z. B. die Seekaufleute von den Konvoischiffen, die ihnen der Staat gegen teures Geld zum Schutz vor Piraten zur Verfügung stellte. Stattdessen versicherten sie ihre Frachten.

Die moderne Verhaltenstheorie (Kahneman 2003) verweist auf unsere begrenzte Rationalität: Wir überschätzen geringe Risiken mit hoher Wahrscheinlichkeit und unterschätzen hohe Risiken mit geringer Wahrscheinlichkeit. Daher sollte der moderne Staat Anreize und Unterstützung zur Versicherung großer Risiken wie Berufs- oder Erwerbsunfähigkeit bieten oder gar dazu verpflichten.

Die moderne Verantwortungsethik (Dworkin 2000) verweist auf die Notwendigkeit gleicher Ressourcenausstattung für die Verbreitung von Risikofreude und Übernahme individueller Verantwortung: Unter dem Schleier des Nicht-Wissens entscheiden sich Individuen für einen Versicherungsvertrag, der gleichzeitig eine generöse Absicherung von Arbeitsmarktrisiken und eine effektive Kontrolle des moralischen Verhaltensrisikos enthält.

Die moderne Finanzwissenschaft (Corneo 2006) beweist, dass eine ex ante Umverteilung von Investitionsrisiken, etwa durch progressive Einkommensteuer, wachstumsfördernde Effekte haben kann.

Die Theorie der institutionellen Komplementarität (Hall/Soskice 2001) weist darauf hin, dass hohe berufliche oder betriebsspezifische Qualifikationen nicht nur eine generöse Arbeitslosenversicherung, sondern auch eine umfassende aktive Arbeitsmarktpolitik voraussetzen.

\subsection{SICHERUNGSANREIZE FÜR INNOVATIVE VERHALTENSRISIKEN}

Vor dem Hintergrund dieser Theorien stellt sich die Frage: Wie könnte das innovative Verhaltensrisiko institutionell gestärkt werden? Diese Frage steckt letztlich auch im Konzept „Flexicurity“, das sich die Europäische Beschäftigungsstrategie auf ihre Fahnen geschrieben hat. ${ }^{10}$ Anders formuliert: Welche neuen Sicherheiten könnten die Risikobereitschaft zur internen wie externen Flexibilität erhöhen?

\begin{tabular}{l}
\hline Ausführlicher und umfassend hierzu Schmid \\
(2008b) und Schmid (2008a), S. 281-328. \\
\hline 8 Das „moralische Risiko “ (in der Fachsprache ,mo- \\
ral hazard') resultiert vor allem aus der Informati- \\
onsasymmetrie zwischen Versicherer und Versi- \\
cherten. Versicherte können ihren Informations- \\
vorsprung in verschiedener Weise auf Kosten der \\
Versichertengemeinschaft ausnutzen, vor allem \\
durch die willentliche Herbeiführung des Risikos, \\
durch Untätigkeit zur Vermeidung des Risikos und \\
durch die Vortäuschung von Aktivitäten oder \\
durch die unbegründete Ablehnung eines Ange- \\
bots (hier eines Arbeitsplatzes oder einer Ausbil- \\
dung) zur Beendigung des Zustandes. \\
\hline 9 Vgl. auch hierzu ausführlicher Schmid (2008b) und \\
Schmid (2008a), S. 213-241 mit weiteren Litera- \\
turverweisen. \\
\hline 10 Zu einer umfassenden und nüchternen Auseinan- \\
dersetzung mit diesem Kunstwort und seinen da- \\
hinter liegenden Konzepten vgl. Keller/Seifert \\
(2008) sowie mehrere Beiträge in Kronauer/Linne \\
(2005).
\end{tabular}


Eine Möglichkeit, das innovative Verhaltensrisiko zu stärken, könnte in einer Teilung der Beiträge bestehen. Dabei würde ein Teil des bisherigen Beitrags zur Arbeitslosenversicherung auf einem persönlichen Entwicklungskonto (PEK) angelegt. Die derzeit günstige Finanzlage der Bundesagentur für Arbeit (BA) hätte als Gelegenheitsfenster genutzt werden können, die Senkung des Beitragssatzes zugunsten von PEK moderater ausfallen zu lassen. Die Mittel zur Verlängerung des Arbeitslosengeldbezugs hätten in ein solches Konto umgelenkt werden können. Diese PEKs sollten aus allgemeinen Steuermitteln in einer Weise ergänzt werden, dass alle Beschäftigten unabhängig von ihrem Beitrag auf den gleichen Kontenstand kommen. PEKs könnten darüber hinaus durch Tarifverträge aufgestockt werden.

Den Beschäftigten werden also Ansprüche gutgeschrieben, ohne dass die verabredeten Summen tatsächlich angespart sind. Der Rückgriff auf die Konten - also das Ziehungsrecht - erfolgt nach politisch festgelegten Regeln. Im Gegensatz zu den individuellen Sparkonten derzeitiger Privatisierungsvorschläge sollen keine „Eigentumsrechte" erworben werden. Werden die PEKs nicht genutzt, verfallen sie am Ende des Erwerbslebens.

Die persönlichen Entwicklungskonten sind als Ergänzung und nicht als Ersatz für den „aktiven“ Bestandteil bisheriger Arbeitsmarktpolitik zu verstehen. In arbeitsmarktpolitisch begründeten Fällen soll Arbeitslosen also nach wie vor mit vermittlungsfördernden Maßnahmen, finanziert aus dem rein solidarischen Beitragstopf, unter die Arme gegriffen werden. Die Entnahmen aus dem PEK sollten der individuellen Entscheidung überlassen bleiben, aber an bestimmte Bedingungen geknüpft werden. Generell sollen die Konten zur Finanzierung des Erhalts oder der Verbesserung der Beschäftigungsfähigkeit dienen; insbesondere sollten sie für Weiterbildung, zum Ausgleich reduzierter Arbeitszeiten und zur Überbrückung geminderter Verdienste verwendet werden. In einem Anfangsschritt könnte das Ziehungsrecht auf die Priorität der beruflichen Weiterbildung eingeschränkt werden. Die Kontrolle der Einhaltung dieser Bedingung könnte über eine Weiterbildungsvereinbarung mit dem entsprechenden Betrieb und der zuständigen Arbeitsagentur erfolgen. Die Gewerkschaften könnten solche Vereinbarungen durch Kollektivverträge vorantreiben.
Selbstverständlich wäre noch eine Fülle von (zum Teil technischen) Einzelfragen der Umsetzung zu klären, um möglichen Missbrauch oder unerwünschte Nebeneffekte unter Kontrolle zu halten. Das betrifft etwa die Frage der Nutzung unausgeschöpfter Konten gegen Ende des Arbeitslebens, wo „Erfindungsreichtum“ (also das moralische Verhaltensrisiko) der arbeitsmarktpolitischen Intention des Instruments (also dem innovativen Verhaltensrisiko) ein Schnippchen schlagen könnte. Zur Kontrolle dieses Problems wäre an eine lineare Diskontierung des Kontos ab dem Alter von 50 Jahren zu denken. Auch die mögliche Verdrängung betrieblich finanzierter Weiterbildungsinvestitionen durch PEKs wäre zu beachten.

Die Anspruchsvoraussetzungen für Ziehungsrechte aus dem persönlichen Entwicklungskonto (PEK) wären die gleichen wie beim Arbeitslosengeld, d.h. eine Mindestbeschäftigungszeit in einer Rahmenfrist. Nach dieser Mindestbeschäftigungszeit kann über das volle PEK verfügt werden. Beitragspflicht und regelgebundener Steuerzuschuss bewirken also in einem maßvollen Umfang sowohl intertemporale (zugunsten der rush hour Erwerbsphase) als auch vertikale (zugunsten geringer Einkommensbezieher und höherer Risikogruppen) Umverteilung.

Der Beitragssatz von einem Prozentpunkt erbrächte derzeit ein Volumen von 7,5 Mrd. € für PEKs. Mit entsprechend ergänzten Steuermitteln könnte das Gesamtvolumen auf 15 Mrd. $€$ aufgestockt werden. Umgerechnet auf 27 Mio. sozialversicherungspflichtige Beschäftigte und eine durchschnittliche Arbeitslebensdauer von 40 Jahren, ergäbe sich ein PEK von etwa $22.000 €$ (Gegenwartswert). Wie bei der derzeitigen Arbeitslosenversicherung wäre eine Ausweitung der Versicherungspflicht auf alle Erwerbstätigen, also auch auf Beamte, Selbstständige und geringfügig Beschäftigte, wünschenswert. Entsprechend würden sich dann auch die hier angeführten Orientierungsdaten verändern.

\section{Die Zukunft der Arbeitsmarktpolitik}

Ob die Idee eines persönlichen Entwicklungskontos als Element zukünftiger Arbeitsmarktpolitik weiterer wissenschaftli- cher Prüfung standhalten und auch politische Mehrheiten finden wird, muss beim derzeitigen Stand der Diskussion offen bleiben. Unabhängig davon gilt, dass moderne Arbeitsmarktpolitik die Orientierung am Lebenslauf, am lebenslangen Lernen und an der Gleichstellung der Geschlechter ernst nehmen muss. Das bedeutet, nicht nur das Einkommensrisiko bei Arbeitslosigkeit, sondern auch die Einkommensrisiken bei kritischen Übergängen im Erwerbsverlauf abzusichern. Nicht nur Arbeit, sondern auch Übergänge sollten sich lohnen.

Moderne Arbeitsmarktpolitik darf sich nicht nur auf die Zähmung des moralischen Verhaltensrisikos konzentrieren. Sie sollte - viel mehr als bisher - das innovative Verhaltensrisiko fördern. Individuen sollten in die Lage versetzt werden, Autoren ihres eigenen Lebens zu werden (Sen 2001). Zur Gleichheit existenzieller Ressourcen wie Wohnung, Gesundheit und Bildung käme die gleiche Befähigung zu einer autonomen Lebensführung als $\mathrm{Ge}$ währleistungsaufgabe des Sozialstaates hinzu.

Dabei würde die Ergänzung der solidarischen Arbeitslosenversicherung durch ein persönliches Entwicklungskonto (PEK) eine entscheidende Rolle spielen. Gegenüber einer bloßen Differenzierung des Arbeitslosengeldbezugs nach Alter hätte sie mehrere Vorteile:

(1) Das PEK würde das Äquivalenzprinzip stärken, denn individuelle Beiträge, ergänzt durch einen solidarisch umverteilenden Steuerbeitrag, entlasten die Beiträge zur Finanzierung "versicherungsfremder" Leistungen. Dadurch tragen sie auch zur Minderung des moralischen Verhaltensrisikos bei.

(2) Des Weiteren stärken PEKs die Gerechtigkeit. Alte haben gegenüber jungen Beschäftigten nicht per se höhere Arbeitsmarktrisiken. Erziehende Mütter oder Väter können von Arbeitslosigkeit härter getroffen werden als Ältere. Da über einen Teil der Beiträge nun je nach Lebenslage im Rahmen eines Regelsystems frei verfügt werden kann, würde auch das Gefühl abgemildert, bei langen Beitragszahlungen „enteignet" zu werden.

(3) Und schließlich fördern PEKs auch die Effizienz. Werden arbeitsmarktpolitische Maßnahmen aus den eigenen Ziehungs- 
rechten mitfinanziert, ist zu erwarten, dass diese Maßnahmen aus Eigeninteresse sorgfältig ausgewählt und hoch motiviert umgesetzt werden. Die Administrierung persönlicher Entwicklungskonten setzt jedoch wegen der begrenzten Information von Individuen eine Modernisierung der Arbeitsmarktdienstleistungen voraus, vor allem eine Stärkung der Arbeitsberatungskapazität der Arbeitsagenturen.
Es entspräche den Prinzipien des modernen Sozialstaats (Kaufmann 2003) und dem modernen Staatsverständnis (Schuppert 2005), die altehrwürdige Arbeitslosenversicherung zu einer Beschäftigungsversicherung zu erweitern. Der Hauptvorteil bestünde vor allem in einer Stärkung des innovativen Verhaltensrisikos. Die Bereitschaft von Arbeitnehmern wie Arbeitgebern würde zunehmen, riskante Investitionsentscheidungen zu treffen. Vor allem würde die Bereitschaft befördert, sich weiterzubilden, mit flexiblen Arbeitszeiten zu arbeiten, zwischenbetriebliche Mobilität zu zeigen oder Selbstständigkeit einzugehen. Eine Beschäftigungsversicherung würde damit nicht nur die ökonomische Wohlfahrt steigern, sondern auch dem sozialpolitischen Ziel einer größeren Selbstbestimmung und einer ausbalancierten Gestaltung der Arbeits- und der Lebenswelt näher kommen.

\section{LITERATUR}

Beck, U. (1986): Risikogesellschaft. Auf dem Weg in eine andere Moderne, Frankfurt a.M

Bernstein, P. L. (1996): Against the Gods. The Remarkable Story of Risk, New York et al.

Bosch, G./Weinkopf, C. (2006): Mindestlöhne in Großbritannien - Ein geglücktes Realexperiment, in: WSI-Mitteilungen 3, S. 125-130 Boss, A./Brown, A./Snower, D. J. (2007): Beschäftigungskonten für Deutschland, Kieler Arbeitspapiere 1325, Institut für Weltwirtschaft an der Universität Kiel

Corneo, G. (2006): New Deal für Deutschland. Der dritte Weg zum Wachstum, Frankfurt/New York

Dworkin, R. (2000): Sovereign Virtue. The Theory and Practice of Equality, Cambridge, MA, and London

Hall, P. A./Soskice, D. (Hrsg.) (2001): Varieties of Capitalism. The Institutional Foundations of Comparative Advantages, Oxford

Ifo-Schnelldienst (2003): Nr. 6 vom 31.März (Sonderausgabe) Kahneman, D. (2003): Maps of Bounded Rationality: A Perspective of Intuitive Judgment and Choice, in: Frangsmyr, T. (Hrsg.): Les Prix Nobel 2002, Stockholm

Kaufmann, F. X. (2003): Varianten des Wohlfahrtsstaats. Der deutsche Sozialstaat im internationalen Vergleich, Frankfurt a. M.

Keller, B./Seifert, H. (2008): Flexicurity: Ein europäisches Konzept und seine nationale Umsetzung, Bonn, Friedrich-Ebert-Stiftung, Expertisen und Dokumentationen

Kronauer, M./Linne, G. (Hrsg.) (2005): Flexicurity. Die Suche nach Sicherheit in der Flexibilität, Berlin

Kull, S./Riedmüller, B. (2007): Auf dem Weg zur Arbeitsmarktbürgerin? Neue Konzepte der Arbeitsmarktpolitik am Beispiel alleinerziehender Frauen, Berlin, Forschung aus der Hans-Böckler-Stiftung, Bd. 84 Martens, D. (2008): Arbeiten mit dem Klebeeffekt, in: Tagesspiegel, 14.05., S. 14

Manning, A. (2003): Monopsony in Motion: Imperfect Competition in Labour Markets, Princeton
Metcalf, D. (2007): Why has the British National Minimum Wage had little or no Impact on Employment?, London School of Economics, CEP Discussion Paper 781, London

Oschmiansky, H. (2007): Der Wandel der Erwerbsformen und der Beitrag der Hartz-Reformen: Berlin und die Bundesrepublik Deutschland im Vergleich, Discussion Paper SP I 2007-104, Wissenschaftszentrum Berlin für Sozialforschung (WZB)

Promberger, M. in Zusammenarbeit mit Bellmann, L./Dreher, Ch./Sowa, F./Schramm, S./Theuer, St. (2006a): Leiharbeit im Betrieb - Strukturen, Kontexte und Handhabung einer atypischen Beschäftigungsform, Nürnberg, Institut für Arbeitsmarkt- und Berufsforschung, Abschlussbericht des Forschungsprojektes HBS-2002-418-3, gefördert von der Hans-Böckler-Stiftung

Promberger M. (2006b): Leiharbeit - Flexibilität und Prekarität in der betrieblichen Praxis, in: WSI-Mitteilungen 5, S. 263-269

Schmid, G. (2002): Wege in eine neue Vollbeschäftigung - Übergangsarbeitsmärkte und aktivierende Arbeitsmarktpolitik, Frankfurt a. M. und New York

Schmid, G. (2008a): Full Employment in Europe - Managing Labour Market Transitions and Risks, Cheltenham, UK and Northampton, MA, USA Schmid, G. (2008b): Von der Arbeitslosen- zur Beschäftigungsversicherung. Wege zu einer neuen Balance individueller Verantwortung und Solidarität durch eine lebenslauforientierte Arbeitsmarktpolitik, Bonn, Gutachten für die Friedrich-Ebert-Stiftung, Projekt Zukunft 2020, Gesprächskreis Arbeit und Qualifizierung

Schulze Buschoff, K. (2007): "Neue Selbstständige“ und soziale Sicherheit - Ein europäischer Vergleich, in: WSI-Mitteilungen 7, S. 387-393 Schuppert, G. F. (Hrsg.) (2005): Der Gewährleistungsstaat - ein Leitbild auf dem Prüfstand, Baden-Baden

Sen, A. (2001): Development as Freedom, New York

Storrie, D. (2002): Temporary Agency Work in the European Union, Dublin, Foundation for the Improvement of Living Conditions

Thiede, R. (2000): Alterssicherung - Modell zur Modernisierung des Rentensystems, in: Soziale Sicherheit 2, S. 48-52 